\title{
Perfil epidemiológico das mortes por câncer de mama e covid-19
}

\author{
Epidemiological profile of deaths from breast cancer and covid-19 \\ Perfil epidemiológico de muertes por cáncer de mama y covid-19
}

Recebido: 16/06/2021 | Revisado: 24/06/2021 | Aceito: 27/06/2021 | Publicado: 12/07/2021

Kely Paviani Stevanato

ORCID: https://orcid.org/0000-0003-1872-8246 Universidade Estadual de Maringá, Brasil

E-mail: kelypaviani@hotmail.com

Amanda de Carvalho Dutra

ORCID: https://orcid.org/0000-0002-2372-7275

Universidade Estadual de Maringá, Brasil

E-mail: amandacarvalhodutra@gmail.com

Lander dos Santos

ORCID: https://orcid.org/0000-0001-7734-2189

Centro Universitário Cesumar, Brasil

E-mail: lander_ds@hotmail.com

Igor Rosckovisk

ORCID: https://orcid.org/0000-0002-9748-1917

Universidade Estadual de Maringá, Brasil

E-mail: igorrosz@gmail.com

Helena Fiats Ribeiro

ORCID: https://orcid.org/0000-0002-6242-0081

Universidade Estadual de Maringá, Brasil

E-mail: helenafiats@hotmail.com

Maria Dalva de Barros Carvalho

ORCID: https://orcid.org/0000-0002-1377-3331

Universidade Estadual de Maringá, Brasil

E-mail: mdbcarvalho@gmail.com

Raíssa Bocchi Pedroso

ORCID: https://orcid.org/0000-0002-0076-1032

Universidade Estadual de Maringá, Brasil

E-mail: raissap@gmail.com

Sandra Marisa Pelloso

ORCID: https://orcid.org/0000-0001-8455-6839

Universidade Estadual de Maringá, Brasil

E-mail: smpelloso@gmail.com

\begin{abstract}
Resumo
Pacientes oncológicos possuem riscos mais elevados para o desenvolvimento da doença por COVID-19 em sua forma mais severa. Objetivo: analisar a mortalidade por câncer de mama associada a COVID-19 em mulheres brasileiras. Metodologia: Trata-se de um estudo quantitativo. Os dados foram coletados no site do Open Data SUS, no período de janeiro a agosto de 2020. Utilizou-se estatística descritiva para análise dos dados. Resultados: o câncer de mama associado ao COVID-19 causou 69 óbitos nesse período, as idades que tiveram maior número de óbitos foram nos intervalos de idade 45-49 (14.5\%), 60-64 (14.5\%) e 65-69 anos (14.5\%). Sobre a raça, (56,52\%) eram brancos, seguida da cor parda $(31,88 \%)$. Apenas $(31,88 \%)$ apresentaram 8 anos ou mais de estudos e $(49.27 \%)$ eram casadas. Em relação a causa básica da morte, o CID B34.2 (Infecção pelo Coronavírus de localização não especificada) se apresentou em maior número, com uma frequência de 52 (75.36\%) e o CID C50.9 (Neoplasia maligna de mama, não especificada) teve uma frequência de $17(24.64 \%)$. A cidade com maior número de óbitos por câncer de mama associada a COVID-19 no qual atingiu (20,29\%) dos casos foi o Rio de Janeiro (RJ), seguida pela cidade de São Bernardo do Campo (SP) com (7.25\%). Conclusão: O aumento da mortalidade por câncer de mama no período da pandemia do COVID-19 no Brasil pode estar atribuído à imunossupressão dessas mulheres e as medidas de enfrentamento ao COVID-19, no qual reduziu a procura por cuidados de saúde, acesso e disponibilidade de serviços de diagnóstico.
\end{abstract}

Palavras-chave: Neoplasias da mama; Mortalidade; Perfil epidemiológico; COVID-19.

\section{Abstract}

Cancer patients are at higher risk for the development of COVID-19 disease in its most severe form. Objective: to analyze mortality from breast cancer associated with COVID-19 in Brazilian women. Methodology: This is a quantitative study. Data were collected on the Open Data SUS website, from January to August 2020. Descriptive statistics were used for data analysis. Results: breast cancer associated with COVID-19 caused 69 deaths in this 
period, the ages with the highest number of deaths were in the age ranges 45-49 (14.5\%), 60-64 (14.5\%) and 65-69 years $(14.5 \%)$. Regarding race, $(56.52 \%)$ were white, followed by brown (31.88\%). Only (31.88\%) had 8 years or more of education and $(49.27 \%)$ were married. Regarding the underlying cause of death, he presented ICD B34.2 (Infection by Coronavirus of unspecified location) in greater numbers, with a frequency of $52(75.36 \%)$ and ICD C50.9 (Malignant breast cancer, unspecified) had a frequency of 17 (24.64\%). The city with the highest number of deaths from breast cancer associated with COVID-19, which reached 14 (20.29\%) of cases, was Rio de Janeiro (RJ), followed by the city of São Bernardo do Campo (SP) with $5(7.25 \%)$. Conclusion: The increase in breast cancer mortality during the COVID-19 pandemic in Brazil can be attributed to the immunosuppression of these women and the COVID-19 coping measures, which reduced the demand for health care, access and availability of diagnostic services.

Keywords: Breast neoplasms; Mortality; Health profile; COVID-19.

\section{Resumen}

Los pacientes con cáncer tienen un mayor riesgo de desarrollar la enfermedad COVID-19 en su forma más grave. Objetivo: analizar la mortalidad por cáncer de mama asociado a COVID-19 en mujeres brasileñas. Metodología: Este es un estudio cuantitativo. Los datos se recopilaron en el sitio web Open Data SUS, de enero a agosto de 2020. Se utilizaron estadísticas descriptivas para el análisis de los datos. Resultados: el cáncer de mama asociado a COVID-19 causó 69 muertes en este período, las edades con mayor número de muertes se ubicaron en los rangos de edad 45-49 (14.5\%), 60-64 (14.5\%) y 65-69 años ( $14,5 \%)$. En cuanto a la raza, $(56,52 \%)$ eran blancos, seguidos de los morenos $(31,88 \%)$. Solo $(31,88 \%)$ tenía 8 años o más de educación y $(49,27 \%)$ estaban casados. En cuanto a la causa subyacente de muerte, presentó la CIE B34.2 (Infección por Coronavirus de ubicación no especificada) en mayor número, con una frecuencia del $52(75,36 \%)$ y la CIE C50.9 (Cáncer maligno de mama, no especificado) tuvo una frecuencia de $17(24,64 \%)$. La ciudad con mayor número de muertes por cáncer de mama asociado a COVID-19, que alcanzó el $14(20,29)$ de los casos, fue Río de Janeiro (RJ), seguida de la ciudad de São Bernardo do Campo (SP) con un 5 (7,25\%). Conclusión: El aumento de la mortalidad por cáncer de mama durante la pandemia COVID-19 en Brasil se puede atribuir a la inmunosupresión de estas mujeres y las medidas de afrontamiento del COVID-19, que redujeron la demanda de atención médica, el acceso y la disponibilidad de servicios de diagnóstico.

Palabras clave: Neoplasias de la mama; Mortalidad; Perfil de salud; COVID-19.

\section{Introdução}

A pandemia COVID-19 e a síndrome respiratória aguda grave Coronavírus 2 (SARS-CoV-2), atingiu proporções alarmantes no mundo pela rápida disseminação dos casos e da mortalidade. De acordo com a Organização Panamericana de Saúde (OPAS) até 04 de janeiro de 2021, foram 1.839.660 mortes e 83.910.386 casos confirmados em todo o mundo (Curigliano, et al., 2020; OPAS, 2020).

A alta incidência dos casos de COVID-19 e sua gravidade têm preocupado autoridades, profissionais e sistema de saúde, principalmente com pessoas pertencentes ao grupo de risco, como idosos, crianças, pessoas com comorbidades como hipertensão, obesidade, diabetes, câncer, entre outras (Dietz, et al., 2020).

Pacientes oncológicos são considerados imunodeprimidos, condição fisiológica da própria doença ou pelo efeito dos imunossupressores relacionados aos tratamentos de quimioterapia. Estas pessoas apresentam riscos mais elevados para o desenvolvimento da doença por COVID-19 em sua forma mais severa, com uma taxa de letalidade de 5,6\% em qualquer neoplasia (SBOC, 2020; Thuler, et al., 2020; Emami, et al., 2020; Salako, et al., 2020).

Estudos têm demonstrado que pessoas em tratamento oncológico tiveram mais dificuldade em dar continuidade aos atendimentos devido a fatores como medo da contaminação com a COVID-19, serviços de atendimento reduzidos, limitação de recursos para a continuidade do tratamento e proteção para equipe e pacientes (Manoj, et al,2020; Olabumuyi, et al, 2020). Vários são os problemas que permeiam estas questões, para mulheres com câncer de mama são a piora do prognóstico geral das mulheres com câncer de mama, com maiores taxas de hospitalização, maior admissão em Unidades de Terapia Intensiva (UTI), uso de ventilação mecânica e aumento da mortalidade pela doença (Elghazawy, et al, 2020; Pardo, et al., 2020). O principal fator associado a dificuldade no tratamento destas pacientes são mudança de rotina, adaptação para o enfrentamento da pandemia, medo da contaminação, isolamento social e dificuldade de acesso aos serviços especializados. Ainda, observa-se a falta de estratégias de direcionamento aos serviços de tratamento do câncer uma vez que foi preciso o redirecionamento de 
leitos exclusivos para acompanhamento de pacientes infectados por COVID-19, sendo estes leitos de enfermaria e leitos de UTI, ocasionando assim uma diminuição de leitos destinados a internamentos para outras patologias (Luther \& Agrawal, 2020).

As repercussões diante destas incertezas para os pacientes são desastrosas, porém, ainda são escassos estudos que mostram o impacto da pandemia em pacientes oncológicos. Os estudos se baseiam em protocolos, revisões, guidelines (Elghazawy, et al., 2020; Chan, et al., 2020). De acordo com estes mesmos autores é preciso gerenciar pacientes com câncer, bem como profissionais especialistas para atender a esse público.

O câncer de mama já é considerado uma doença com alta taxa de morbidade e mortalidade em mulheres em todo o mundo e, este cenário pode estar se agravando diante deste novo normal que estamos vivenciando pela pandemia do COVID19. De fato, a pandemia incidiu drasticamente no tratamento do câncer forçando uma mudança na prática de atendimento no sentido de proteger pacientes e profissionais (Luther \& Agrawal, 2020).

Atualmente, o conhecimento do perfil epidemiológico e do tratamento da doença COVID-19 é alvo de muitos estudos, porém, poucos estudos foram encontrados na literatura que relacionam esta doença com o câncer de mama, a maioria discute manejo da prática (Luther \& Agrawal, 2020) e guidelines (Manoj, et al ,2020). Diante desta situação, e do desconhecimento dos efeitos da COVID-19 em pacientes oncológicos, no que diz respeito tanto a sobrevida como a mortalidade e a falta de literatura científica com essa temática, este estudo tem como objetivo analisar a mortalidade por câncer de mama associada a COVID-19 em mulheres brasileiras.

\section{Metodologia}

Estudo descritivo, de abordagem quantitativa, realizado com dados públicos. Os dados sobre mortalidade foram coletados no site do Open Data SUS (https://opendatasus.saude.gov.br/) e refere a mortalidade por COVID-19 associado a câncer de mama em mulheres brasileiras no período de janeiro a agosto de 2020.

Para a coleta dos dados foi utilizado, a Classificação Internacional de Doenças CID 10 onde B34.2, corresponde a "Infecção por coronavírus de localização não especificada", no campo causa básica e os CID C50 "Neoplasia maligna da mama", C50.0 "Neoplasia maligna do mamilo e aréola", C50.1 "Neoplasia maligna da porção central da mama", C50.2 "Neoplasia maligna do quadrante superior interno da mama", C50.3 "Neoplasia maligna do quadrante inferior interno da mama", C50.4 "Neoplasia maligna do quadrante superior externo da mama", C50.5 "Neoplasia maligna do quadrante inferior externo da mama", C50.6 "Neoplasia maligna da porção axilar da mama", C50.8 "Neoplasia maligna da mama com lesão invasiva”,C50.9 "Neoplasia maligna da mama, não especificada”, U07.1"Vírus identificado - atribuído a um diagnóstico de COVID-19 confirmado por testes laboratoriais e U07.2 "vírus não-identificado - inclui diagnóstico de caso provável ou caso suspeito de COVID-19 no campo atestado, ambos contidos no Sistema de Informação sobre Mortalidade (SIM), em um segundo momento, foi utilizado os CID C50.0, C50.1, C50.2, C50.3, C50.4, C50.5, C50.6, C50.8, C50.9, como causa básica e os CID B34.2, U07.1 e U07.2 no campo atestado.

Os dados foram tabulados e analisados de forma descritiva no software Microsoft Excel 2019 e posteriormente analisados pelo software Qgiz versão 3.10.8 para a realização do geoprocessamento, no qual identificou no mapa os Estados de residência das mulheres que foram a óbito por estas doenças no país.

\section{Resultados}

O número total de óbitos de mulheres brasileiras por câncer de mama no período de 01 de janeiro a 15 de maio de 2020 foi de 5.522, uma diferença de 336 óbitos por esta doença em relação ao mesmo período do ano passado (2019) que atingiu o total de 5.186 óbitos. Dos 336 óbitos, 69 (20,54\%) foram de câncer de mama associado a COVID-19. As idades que 
tiveram maior número de óbitos foram nos intervalos de idade 45-49 (14.5\%), 60-64 (14.5\%) e 65-69 anos (14.5\%). A raça/cor branca foi predominante $39(56,52 \%)$ seguida da cor parda $22(31,88 \%)$, em relação a escolaridade, $22(31,88 \%)$ apresentaram de 8 a 11 anos de estudos, seguidos por 1 a 3 anos (20.30\%) e 4-7 anos (20.30\%) e, 34 mulheres (49.27\%) eram casadas (Tabela1).

Tabela 1 - Características demográficas dos óbitos em mulheres por câncer de mama e COVID-19 (n=69), 2020.

\begin{tabular}{|c|c|c|}
\hline Variáveis & Número (n) & Frequência $(\%)$ \\
\hline \multicolumn{3}{|l|}{ Idade } \\
\hline $30-34$ & 1 & $1.44 \%$ \\
\hline $35-39$ & 1 & $1.44 \%$ \\
\hline $40-44$ & 2 & $2.90 \%$ \\
\hline $45-49$ & 10 & $14.50 \%$ \\
\hline $50-54$ & 3 & $4.35 \%$ \\
\hline $55-59$ & 9 & $13.04 \%$ \\
\hline $60-64$ & 10 & $14.50 \%$ \\
\hline $65-69$ & 10 & $14.50 \%$ \\
\hline $70-74$ & 7 & $10.14 \%$ \\
\hline $75-79$ & 5 & $7.24 \%$ \\
\hline $80-84$ & 3 & $4.35 \%$ \\
\hline $85-89$ & 3 & $4.35 \%$ \\
\hline $90-94$ & 2 & $2.90 \%$ \\
\hline $95-99$ & 3 & $4.35 \%$ \\
\hline \multicolumn{3}{|l|}{ Raça/Cor } \\
\hline Branca & 39 & $56.52 \%$ \\
\hline $\begin{array}{l}\text { Preta } \\
\text { Amarela }\end{array}$ & 6 & $8.70 \%$ \\
\hline Parda & 1 & $1.45 \%$ \\
\hline \multirow[t]{2}{*}{ N/A } & 22 & $31.88 \%$ \\
\hline & 1 & $1.45 \%$ \\
\hline \multicolumn{3}{|l|}{ Escolaridade } \\
\hline Nenhuma & 1 & $1.45 \%$ \\
\hline 1-3 anos & 14 & $20.30 \%$ \\
\hline 4 a 7 anos & 14 & $20.30 \%$ \\
\hline 8 a 11 anos & 22 & $31.88 \%$ \\
\hline 12 e mais & 10 & $14.49 \%$ \\
\hline $\mathrm{N} / \mathrm{A}$ & 7 & $10.14 \%$ \\
\hline $\mathrm{N} / \mathrm{R}$ & 1 & $1.44 \%$ \\
\hline \multicolumn{3}{|l|}{ Estado Civil } \\
\hline Solteira & 15 & $21.74 \%$ \\
\hline Casada & 34 & $49.27 \%$ \\
\hline Viúva & 10 & $14.49 \%$ \\
\hline Separada & 4 & $5.80 \%$ \\
\hline União consensual & 4 & $5.80 \%$ \\
\hline $\mathrm{N} / \mathrm{A}$ & 2 & $2.90 \%$ \\
\hline
\end{tabular}

Fonte: Ministério da Saúde - OPEN DATASUS. 
Em relação as causas dos óbitos, somente o CID C50.9 (Neoplasia maligna de mama, não especificada) foi encontrado nas declarações de óbito em qualquer linha. A causa básica da morte que corresponde a doença ou lesão que iniciou a cadeia de acontecimentos patológicos no qual levaram a morte, apresentou o CID B34.2 (Infecção pelo Coronavírus de localização não especificada) em maior número, com uma frequência de $75.36 \%$ (52), e o CID C50.9 (Neoplasia maligna de mama, não especificada) teve uma frequência de 24.64\% (17) (Tabela 2).

$\mathrm{Na}$ linha A da declaração de óbito no qual corresponde a causa imediata ou terminal, os CID B34.2 (Infecção pelo Coronavírus de localização não especificada), U07.2 (COVID-19, vírus não identificado, clínico epidemiológico) e U04.9 (Síndrome respiratória aguda grave - SARS/SRAG) teve maior frequência 21.16\% (11), seguido pelos CID B34.2 (Infecção pelo Coronavírus de localização não especificada) e U07.1 (COVID-19, vírus identificado) 13,46\% (7) quando a causa básica foi o CID B34.2 (Infecção pelo Coronavírus de localização não especificada). Já na causa básica CID C50.9 (Neoplasia maligna de mama, não especificada), os CID B34.2 isolado, B34.2 e U04.9, C50.9 isolado, J96.9 (Insuficiência respiratória não especificada) tiveram a mesma frequência 17.65\% (3), seguido pelo CID A41.9 (Septicemia não especificada) $11,76 \%$ (2) (Tabela 2).

$\mathrm{Na}$ linha $\mathrm{b}$ que corresponde a causa intermediária do óbito, ao analisar como causa básica do óbito o CID B34.2, os CID B34.2 e U07.2 foram mais frequentes 13.46\% (7), seguidos pelos CID B34.2 e U07.1 com uma frequência de 7.69\% (4). Na causa básica do óbito C50.9 os CID mais frequentes foram B34.2 isolado 23.54\% (4), seguido por B34.2 e U04.9, C50.9 isolado, J18.9 (Pneumonia não especificada), J96.9 (Insuficiência respiratória não especificada), todos com uma frequência de $11.76 \%$ (2) (Tabela 2).

Ao analisar a tabela 2, a linha c, no qual corresponde a causa intermediária do óbito, encontramos como causas mais frequentes os CID B34.2 e UO7.2 correspondendo a 7.69\% (4), seguido pelos CID B34.2 e U07.1, C50.9, ambos com 5.78\% (3), na causa básica do óbito B34.2. O CID J11.8 (Influenza (gripe) com outras manifestações, devida a vírus não identificado) foi o mais prevalente $11.76 \%$ (2) quando a causa básica foi C50.9. E, 42,33\% (22) dos óbitos descritos na linha II com o CID C50.9 contribuíram para a morte quando a causa básica foi B34.2.

Tabela 2 - Classificação Internacional das Doenças (CID) por linha, de acordo com a declaração de óbito (n=69), 2020.

\begin{tabular}{|c|c|c|c|c|c|c|}
\hline & \multirow{2}{*}{$\begin{array}{c}\text { Causa Básica } \\
\text { B342 } \\
\text { (n. 52) }\end{array}$} & \multirow[b]{2}{*}{ n. } & \multirow[b]{2}{*}{$\%$} & \multicolumn{3}{|l|}{ Causa Básica } \\
\hline & & & & $\begin{array}{l}\text { C50.0 a C50.9 } \\
\text { (n. 17) }\end{array}$ & n. & $\%$ \\
\hline \multirow[t]{13}{*}{ Linha a } & A419 & 5 & $9.62 \%$ & A419 & 2 & $11.76 \%$ \\
\hline & B342 & 3 & $5.77 \%$ & B342 & 3 & $17.65 \%$ \\
\hline & B342, J118 & 1 & $1.92 \%$ & B342, J118 & 0 & - \\
\hline & B342, U049 & 0 & - & B342, U049 & 3 & $17.65 \%$ \\
\hline & B342, U071 & 7 & $13.46 \%$ & B342, U071 & 1 & $5.88 \%$ \\
\hline & B342, U071, A419, J129 & 1 & $1.92 \%$ & B342, U071, A419, J129 & 0 & - \\
\hline & B342, U072 & 5 & $9.62 \%$ & B342, U072 & 0 & - \\
\hline & B342, U072, U049 & 11 & $21.16 \%$ & B342, U072, U049 & 0 & - \\
\hline & C509 & 0 & - & C509 & 3 & $17.65 \%$ \\
\hline & $\mathrm{J} 80 \mathrm{X}$ & 2 & $3.85 \%$ & $\mathrm{~J} 80 \mathrm{X}$ & 0 & - \\
\hline & J80X, U049 & 4 & $7.69 \%$ & J80X, U049 & 1 & $5.88 \%$ \\
\hline & J960 & 7 & $13.46 \%$ & J960 & 0 & - \\
\hline & J969 & 4 & $7.69 \%$ & J969 & 3 & $17.65 \%$ \\
\hline
\end{tabular}




$\begin{array}{ll} & \text { J988 } \\ & \text { R688 } \\ \text { Total } & -* \\ \text { Linha b } & \text { B342 } \\ & \text { B342, U049 } \\ & \text { B342, U071 } \\ & \text { B342, U072 } \\ & \text { B342, U072, C509 } \\ & \text { B342, U072, J189 } \\ \text { C509 } & \text { D70X } \\ \text { J129 } & \text { J180 } \\ \text { J189 } \\ \text { J80X } \\ \text { J81X } \\ \text { J960 } \\ \text { J969 } \\ \text { J969, J189, C509 } \\ \text { U049 } \\ -*\end{array}$

Total

$\begin{array}{rrlrr}1 & 1.92 \% & \text { J988 } & 0 & - \\ 0 & - & \text { R688 } & 1 & 5.88 \% \\ 1 & 1.92 \% & -* & 0 & - \\ \mathbf{5 2} & \mathbf{1 0 0 \%} & & 17 & 100 \%\end{array}$

$\begin{array}{ll}\text { Linha c } & \text { A418 } \\ & \text { B342 } \\ \text { B342, U071 } \\ \text { B342, U072 } \\ \text { C509 } \\ \text { C509, C780 } \\ \text { J118 } \\ \text { U072 } \\ -*\end{array}$

Total

$\begin{array}{ll}\text { Linha d } & \text { B342, U049 } \\ & \text { C509 } \\ \text { E149, I10X } \\ \text { I509 } \\ -*\end{array}$

$\begin{array}{rrl}0 & - & \text { B342 } \\ 1 & 1.92 \% & \text { B342, U049 } \\ 4 & 7.69 \% & \text { B342, U071 } \\ 7 & 13.46 \% & \text { B342, U072 } \\ 2 & 3.85 \% & \text { B342, U072, C509 } \\ 1 & 1.92 \% & \text { B342, U072, J189 } \\ 2 & 3.85 \% & \text { C509 } \\ 1 & 1.92 \% & \text { D70X } \\ 1 & 1.92 \% & \text { J129 } \\ 2 & 3.85 \% & \text { J180 } \\ 3 & 5.78 \% & \text { J189 } \\ 1 & 1.92 \% & \text { J80X } \\ 1 & 1.92 \% & \text { J81X } \\ 1 & 1.92 \% & \text { J960 } \\ 0 & - & \text { J969 } \\ 1 & 1.92 \% & \text { J969, J189, C509 } \\ 1 & 1.92 \% & \text { U049 } \\ 23 & 44.24 \% & -* \\ \mathbf{5 2} & \mathbf{1 0 0 \%} & \end{array}$

$\begin{array}{rr}4 & 23.54 \% \\ 2 & 11.76 \% \\ 0 & - \\ 0 & - \\ 0 & - \\ 0 & - \\ 2 & 11.76 \% \\ 0 & - \\ 0 & - \\ 0 & - \\ 2 & 11.76 \% \\ 1 & 5.88 \% \\ 0 & - \\ 0 & - \\ 2 & 11.76 \% \\ 0 & - \\ 0 & - \\ 4 & 23.54 \% \\ \mathbf{1 7} & \mathbf{1 0 0 \%}\end{array}$


Total

$\begin{array}{ll}\text { Linha II } & \text { C260, C509, I499, I619 } \\ \text { C509 } \\ \text { C509, B342, U072 } \\ \text { C509, C780 } \\ \text { C509, C780, E149 } \\ \text { C509, C786 } \\ \text { C509, C787 } \\ \text { C509, C798 } \\ \text { C509, C80X } \\ \text { C509, C80X, I509 } \\ \text { C509, D589 } \\ \text { C509, E106 } \\ \text { C509, I10X } \\ \text { C509, J449 } \\ \text { C509, J869 } \\ \text { C509, N19X } \\ \text { C798, C795, C787, C509 } \\ \text { I10X, C509 } \\ \text { I10X, E149 } \\ \text { I10X, E149, C449, C509 } \\ \text { I48X } \\ \text { I639, C509 } \\ \text { M069, C509 } \\ \text { U049, C509, I10X } \\ \text {-* }\end{array}$

Total
52

$100 \%$

$1 \quad 1.92 \% \quad \mathrm{C} 260, \mathrm{C} 509, \mathrm{I} 499, \mathrm{I} 619$

22

$42.33 \%$

C509

- C509, B342, U072

$3.85 \% \mathrm{C} 509, \mathrm{C} 780$

$1.92 \%$ C509, C780, E149

$1.92 \% \quad \mathrm{C} 509, \mathrm{C} 786$

$1.92 \% \quad \mathrm{C} 509, \mathrm{C} 787$

$1.92 \% \quad \mathrm{C} 509, \mathrm{C} 798$

$1.92 \%$ C509, C80X

$1.92 \%$ C509, C80X, 1509

- C509, D589

$1.92 \% \quad \mathrm{C} 509, \mathrm{E} 106$

$3.85 \%$ C509, I10X

$1.92 \% \quad \mathrm{C} 509, \mathrm{~J} 449$

$1.92 \% \quad \mathrm{C} 509, \mathrm{~J} 869$

- C509, N19X

$1.92 \% \mathrm{C} 798, \mathrm{C} 795, \mathrm{C} 787, \mathrm{C} 509$

$1.92 \%$ I10X, C509

$3.85 \%$ I10X, E149

$1.92 \%$

$\mathrm{I} 10 \mathrm{X}$

- I48X

$1.92 \% \quad$ I639, C509

$1.92 \%$ M069, C509

0 - U049, C509, I10X

$9 \quad 17.32 \quad-*$

52
$17 \quad 100 \%$

$23.54 \%$

$1 \quad 5.88 \%$

0

0

0

0

0

0

0

$15.88 \%$

0

0

$15.88 \%$

0

$1 \quad 5.88 \%$

0

$1 \quad 5.88 \%$

0

0

$1 \quad 5.88 \%$

0

0

$1 \quad 5.88 \%$

$6 \quad 35.30 \%$

$17 \quad 100 \%$

*Campo não informado

Fonte: Ministério da Saúde - OPEN DATASUS.

Os óbitos estão distribuídos nos estados brasileiros de acordo com o local de residência da mulher, como mostra o Mapa 1, sendo o Rio de Janeiro (RJ) a cidade com maior número de óbitos por câncer de mama associada a COVID-19 no qual atingiu 20,29\% (14) dos casos, seguida pela cidade de São Bernardo do Campo (Estado de São Paulo) com 7.25\% (5) dos óbitos pelas doenças. Ao comparar os Estados com maior número de casos de óbito o Rio de Janeiro e São Paulo obtiveram o mesmo número de casos, correspondendo cada um a 30,43\% (21) dos óbitos. 
Mapa 1. Distribuição dos óbitos por COVID-19 e câncer de mama por estado, Brasil, 2020.

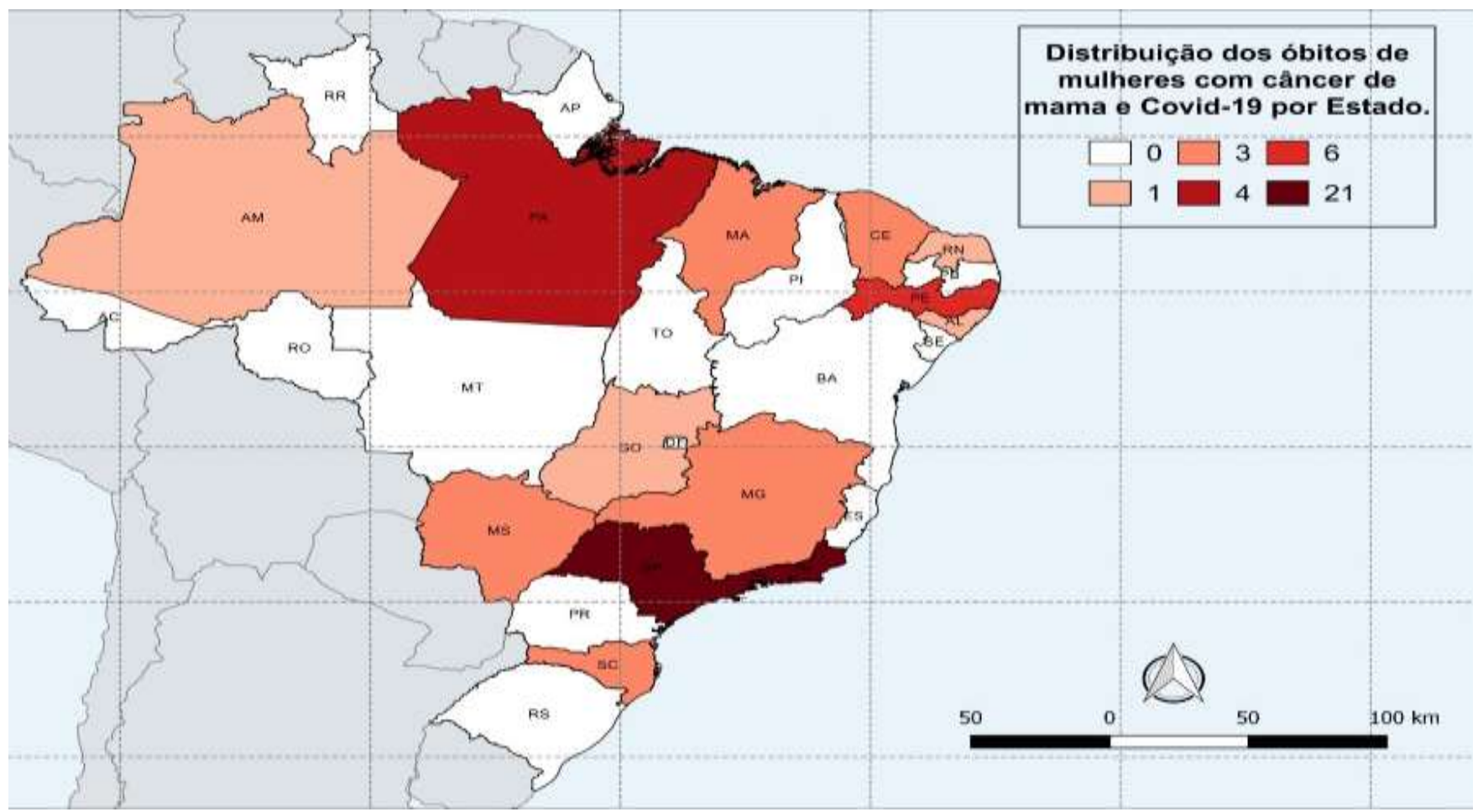

Fonte: Ministério da Saúde - OPEN DATASUS;

\section{Discussão}

A pandemia do COVID-19 incidiu drasticamente no tratamento do câncer, forçando uma mudança na prática de atendimento como também no perfil epidemiológico da doença. Atualmente, muitas pesquisas estudam o conhecimento do perfil epidemiológico e tratamento do COVID-19, porém, poucos estudos foram encontrados na literatura que relacionam esta doença com o câncer de mama.

No Brasil, o número de óbitos analisados em 2020 foi maior comparando com o mesmo período do ano anterior (período sem pandemia). O aumento de óbitos foi de 6,48\% (336) e destes 20,54\% (69) eram de pacientes com câncer de mama que se infectaram por COVID-19. Um dado semelhante foi encontrado em um estudo realizado no Reino Unido que estimou um aumento de $6 \%$ no número de mortes por câncer de mama no período da pandemia, e atribui esse aumento como consequência das medidas nacionais adotadas no período da pandemia do COVID-19, que reduziu a procura por cuidados de saúde, acesso e disponibilidade de serviços de diagnóstico, porém, os autores não associam as duas doenças (Maringe, et al., 2020). Entretanto, um estudo realizado nos Estados Unidos, Canadá e Espanha concluiu que houve associação entre câncer de mama e COVID-19 com outros fatores de riscos gerais (Kuderer, et al., 2020).

No presente estudo pode-se observar uma frequência significativa de óbitos na faixa etária de 40-49 anos, considerada esta uma população de mulheres jovens, no entanto, não podemos afirmar que a infecção pelo Sars-Cov-2 antecipou ou não o prognóstico desfavorável dessas mulheres. Estudo de Liang et al., (2020) na China, com análise nacional de pacientes infectados por Sars-Cov-2 e câncer como comorbidade observou que pacientes com câncer tinham um maior risco de desenvolver eventos graves e piora do quadro. Estudo de Guan, et al., (2020) observou a história de malignidade como fator de risco para desfechos de admissão à UTI ou utilização de ventilação mecânica invasiva ou óbito. Estes estudos analisaram todos os tipos de câncer em pacientes infectados pela doença COVID-19.

O CID B34.2 (Infecção pelo Coronavírus de localização não especificada) foi a causa básica dos óbitos com maior frequência neste estudo. Este resultado demonstra que a doença coronavírus desencadeou uma sucessão de eventos patológicos 
ocasionando a morte dessas mulheres. Apesar disso, as declarações de óbitos também trazem nas linhas "a" e "b" o CID B34.2 associados a outros CID como U07.2 e U04.9 como causa de morte intermediária, ou seja, como consequência. O Ministério da Saúde (MS) em seu manual de Orientações para o preenchimento da Declaração de Óbito no contexto da COVID-19 orienta que o registro da COVID-19 deve ser feito na parte I da declaração de óbito, respeitando à ordenação da cadeia de causas. A causa básica deve estar contida na última linha do atestado e as causas sequenciais, decorrentes da causa básica, devem ser registradas nas linhas acima, linha c, linha b e linha a. Neste mesmo manual orienta-se que na parte II da declaração de óbito devem estar descritas as comorbidades e, no estudo pode-se perceber que a utilização do código C50.9 foi utilizado com maior frequência na parte II.

Este estudo tem como limitação a disponibilidade de dados mais atuais referente a mortalidade no ano de 2020 no Brasil, como também, informações referentes a tempo de tratamento do câncer de mama e período de internação, a fim de comparar a contaminação pelo COVID-19 no prognóstico da doença.

\section{Conclusão}

Os resultados deste estudo mostram que houve um aumento da mortalidade por câncer de mama no período da pandemia do COVID-19 no Brasil, e que este aumento pode estar atribuído em alguns casos à infecção dessas mulheres pela doença COVID-19, no qual pode-se obter um desfecho desfavorável devido a imunossupressão dessas mulheres.

Outro fator que pode ter contribuído para o aumento da mortalidade por câncer de mama neste período foi as medidas nacionais adotadas para o enfrentamento do COVID-19, no qual reduziu a procura por cuidados de saúde, acesso e disponibilidade de serviços de diagnóstico.

Em função das estratégias de vacinação adotadas pelo Governo Federal do Brasil em 17 de janeiro de 2021, recomenda-se para trabalhos futuros o levantamento de dados acerca dos óbitos por câncer de mama em pacientes imunizados pela vacina contra o COVID-19 e a comparação das taxas nos períodos pré e pós-pandemia.

\section{Referências}

Azambuja, E., Trapani, D., Loibl, S., Delaloge, S., Senkus, E., et al. (2020). Management and treatment adapted recommendations in the COVID-19 era: Breast Cancer. ESMO Open.10.1136/esmoopen-2020-000793.

Curigliano, G., Cardoso, M. J., Poortmans, P., Gentilini, O., Pravettoni, G., et al. (2020). Recommendations for triage, prioritization and treatment of breast cancer patients during the COVID-19 pandemic. The Breast, 52 (8), 8-16. 10.1016 / j.breast.2020.04.006

Dietz, J. R., Moran, M. S., Isakof, S. J., Kurtzman, S. H., Willey, S. C., et al. (2020) Recommendations for prioritization, treatment, and triage of breast cancer patients during the COVID-19 pandemic. The Breast, 181, 487-497. 10.1007 / s10549-020-05644-Z

Elghazawy, H., Bakkach, J., Zaghloul, M. S., Abusanad, A., Hussein, M. M., Alorabi, M., et al. (2020) Implementation of breast cancer continuum of care in low- and middle-income countries during the COVID-19 pandemic. Future Oncol, 16 (31), 2217.10.2217/fon-2020-0574

Emami, A., Javanmardi, F., Pirbonyeh, N., Akbari, A. (2020). Prevalence of underlying diseases in hospitalized patients with COVID-19:a systematic review and meta-analysis.Archives of Academic Emergency Medicine, 8 (1): e35. https://www.ncbi.nlm.nih.gov/pmc/articles/PMC7096724/pdf/aaem-8-e35.pdf17.

Guan, W. J., Liang, W. H., Zhao, Y., Liang, H. R., Chen, Z. S., et al. (2020).Comorbidity and its impact on 1590 patients with COVID-19 in China: a Nationwide analysis. European Respiratory Journal, 55 (5), 2000547.10.1183 / 13993003.00547-2020.

Kuderer, N., Choueiri, T. K., Shah, D. P., Shyr, Y., Rubistein, S. M., et al. (2020). Clinical impact of COVID-19 on patient with cancer (CCC19): a cohort study. The Lancet, 395 (10241), 1907-1918.10.1016/S0140-6736(20)31187-9.

Liang, W., Guan, W., Chen, R., Wang, W., Li, J., et al. (2020).Cancer patients in SARS-CoV-2 infection: a Nationwide analysis in China. The Lancet, 21 (3), 30096-6.0.1016/S1470-2045(20)30096-6.

Luther, A., Agrawal A. (2020). A practical approach to the management of breast cancer in the COVID-19 era and beyond. Ecancer medical Science, 14: 1059. 10.3332 / ecancer.2020.1059

Manoj, G. S., Kabeer, K. K., Jafferbhoy, S., Marla, S., Soumian, S., Misra, V., et al. (2020). Breast Cancer Management Guidelines During COVID-19 Pandemic. Indian J Surg, 2, 1-8. 10.1007/s12262-020-02466-7 
Research, Society and Development, v. 10, n. 8, e27210817269, 2021

(CC BY 4.0) | ISSN 2525-3409 | DOI: http://dx.doi.org/10.33448/rsd-v10i8.17269

Maringe, C., Spicer, J., Morris, M., Purushotham, A., Nolte, H., et al. (2020) The impact of the COVID-19 pandemic on cancer deaths due to delays in diagnosis in England, UK: a national, population-based, modelling study. The Lancet Oncology, 21(8), 1023-1034.10.1016 / S1470-2045 (20) 30388-0

Ministério da Saúde (MS). Secretaria de Vigilância em Saúde. Orientações para o preenchimento da Declaração de Óbito no contexto da COVID-19. (2020). http://docs.bvsalud.org/biblioref/2020/05/1096249/orienta-es-para-delcara-o-de-bito.pdf.

Novel Coronavirus Pneumonia Emergency Response Epidemiology Team. (2019) Analysis of the epidemiological characteristics of new coronavirus pneumonia. Chinese Journal of Epidemiology, 41(2), 145-51.https://doi.org/10.3760/cma.j.issn.0254-6450.2020.02.003

Olabumuyi, A. A., Ali-Gombe, M., Biyi-Olutunde, O. A., Gbolahan, O., Iwuji, C. O., Joseph, A. O., et al. (2020). Oncology practice in the COVID-19 pandemic: a reporto $\mathrm{f}$ a Nigerian expert panel discussion (oncology care in Nigeria during the COVID-19 pandemic). Pan Afr Med, 6(36), 153.10.11604/pamj.2020.36.153.23662

Organização Mundial da Saúde (OMS). Coronavirus disease (COVID-19) pandemic. Numbers at a glance.(2020) https://www.who.int/emergencies/diseases/novel-coronavirus 2019?gclid=CjwKCAjw4rf6BRAvEiwAn2Q76jTwPLGB5ONXS dFZenPy0fEvG1m6V9arDD mCvjpQrxR0gtb1MZelkhoCmUsQAvD_BwE.

Pardoa, R., Algara, M., Montero-Fernández, A. M., Sanz, X., Vernet, M., et al. (2020) Diagnosis and locoregional treatment of patients with breast cancer during the COVID-19 pandemic. Revista de Senología Y Patología Mamaria, 33(2):61-67. https://doi.org/10.1016/j.senol.2020.04.002

Salako, O., Okunade, K., Allsop, M., Habeebu, M., Toye, M., et al. (2020). Upheaval in cancer care during the COVID-19 outbreak. Ecancer medical science, 14(97).https://doi.org/10.3332/ecancer.2020.ed97

Sociedade Brasileira de Oncologia Clínica (SBOC). Posicionamento SBOC. (2020). https://sboc.org.br/images/Infogr\%C3\%A1fico_-_corona_v6.pdf.

Thuler, L. C. S., Melo, A. C. (2020).Sars-CoV-2/COVID-19 em pacientes com câncer. Revista Brasileira de Cancerologia, 66(2): e-00970. 10.32635/21769745.RBC.2020v66n2.970.

Chan JJ, Sim Y, Ow SGW, Lim JSJ, Kusumawidjaja G, Zhuang Q, Wong RX, Wong FY, Tan VKM, Tan TJ. (2020). The impact of COVID-19 on and recommendations for breast cancer care: the Singapore experience. Endocr Relat Cancer, 27(9), 307-327.10.1530/ERC-20-0157. PMID: 32544879. 